\title{
Bulk versus surface state contributions to the efficiency of perovskite titanate photoanodes
}

\author{
L. GOMATHI DEVI, T R N KUTTY ${ }^{\dagger}$ and M AVUDAITHAI \\ Department of Inorganc and Physical Chemistry,, Also of Materaals Research Laboratory, \\ Indian Institute of Science, Bangalore 560012 . India
}

\begin{abstract}
Ibstract. Altempt have been made $t$ conhance the photon efficioncy of perovskite titanate electrodes during band gatp illumination by increasing the bulk delectric constant with the substitution of sutatole isovalent ions in the latice so that the Curie point is shifted to room immerature and by decreasing the $N_{i}$, values with controlled chemeal reduction. The photuresponse showed only marganal improvements with these changes in the bulk characleristis. In comparison, the efficiency ncreased considiably when the electrode surfaces are treated by exposing to dilute acids. particularly $\mathrm{HNO}_{3}+\mathrm{HF}$, for extended periods of tume. This is accompanied by the changes in $l_{\text {in }}$ to cathodic direction, red shift in the spectral dependence of photoresponse and marginal decrease of $N_{b}$ in the space charge layer. X-ray photoclectron spectra show that the treated electrode surfaces are not uniform with respeci to oxygen titinum ratios, hydroxyl and fluonde incorporated. The enhanced photon efficiency arses from the combined effect of eliminating the undesirable electron recombination centres in the space charge laver and the presence of heterogeneous surface region- leading to non-untorm potentral distributun ncar the electrode surface. The results also pont in the limitations of applying Schotiky barrier model for semiconducior; electrolyte interface with higher concentrations of surfice states.
\end{abstract}

Keywords. Perovskite tilanates; phetoanodes: surfice states: photun efficiency.

\section{Introduction}

The most popular model for the electrochemistry of semiconductors is the concept that semiconductor electrode/electrolyte interface closely resembles a Schottky barrier formed at metal/semiconductor or semiconductor/semiconductor junctions (Gerischer 1960. 1970; Butler 1977). Assuming that the electrolyte is comparable to an amorphous semiconductor (Williams and Nozik 1984), differences in Fermi levels of the semiconductor and that of the electrolyte can be visualized. At equilibrium the Fermi levels are equalized leading to band bending. The height of the Schottky barrier so generated depends upon the relative positions of the two Fermi levels before equilibration. Accordingly, the potential drop inside the semiconductor varies with the externally applied potential whereas the potential drop in the electrolyteside of the interface i.e. the Helmholtz layer, remains independent of the applied potential. However, this is greatly dependent on the limiting number of surface states (Green 1959). At detectable concentrations of surface states, the changes in potential will be predominant in the Helmholtz layer than in the space charge region, necessitating suitable modifications in the Schottky barrier model, taking into account the potential barrier in the solution-side of the interface (Khan and Bockris 1983). The reason for such a situation is that transfer of electron (or holes) from the energy band states of the semiconductor to the discrete orbitals of the ions in the electrolyte is a charge transfer reaction. This is an activated process and not a conductive flow of charges as of metal/semiconductor interface (Bockris and Khan 1985). The corresponding disparity prevails even when the problem of numerical determination of Fermi levels in the electrolyte can be solved (Gerischer and Ekardt 1983). 
In order to understand the most crucial parameters that may enhance the photoresponse and thereby verify the validity of Schottky barrier model, we have presently adopted two independent approaches: (i) alter the bulk characteristics so as to increase the depletion layer width and (ii) modify the surface characteristics of the electrode fabricated from a given bulk cornposition. The photocurrent flowing through a semiconductor is proportional to the carriers generated by photons within the depletion layer and also the carriers photogenerated in the bulk that diffuse into the depletion layer. The combined efficiency is given by the well-known relation (Butler 1977).

$$
\eta=I_{p} / q I_{0}=1-[\exp (-x W)] /\left(1+\alpha L_{p}\right),
$$

where $I_{p}$ is the photocurrent density, $q$ the electronic charge, $I_{0}$ the photon flux, $\alpha$ the optical absorption coefficient and $L_{p}$ is the hole diffusion length. The depletion layer width is denoted by

$$
W=W_{0}\left(V-V_{\mathrm{fb}}\right)^{1 / 2}
$$

where $W_{0}$ is the width of the depletion layer for $\left(V-V_{\mathrm{fb}}\right)=1$ across it such that

$$
W_{0}=\left(2 \varepsilon \varepsilon_{0} / q N_{D}\right)^{1 / 2},
$$

with $\varepsilon$ the bulk dielectric constant, $\varepsilon_{0}$ the permittivity of vacuum, $N_{D}$ the carrier concentration, $V_{\mathrm{fb}}$ the flat band potential and $V$ the applied potential. For wide band gap semiconductors, $L_{p} \ll W$ and hence the photocurrent is primarily due to the carriers generated in the depletion layer which means that enhanced $W$ should improve the photon efficiency. This can be achieved either by decreasing the carrier concentration or by increasing the effective dielectric constant. $N_{D}$ values in titanates of a given composition can be altered with the degree of chemical reduction i.e. by changing the oxygen partial pressure $\left(p_{\mathrm{O}_{2}}\right)$ in the surrounding atmosphere and the annealing temperature. It is possible to enhance $\varepsilon$-values for perovskite titanates with suitable substitution of isovalent ions in the lattice so that the ferroelectric Curie point $\left(T_{c}\right)$ is shifted to room temperature.

The surface characteristics of titanate electrodes can be modified by exposing them to dilute acids for extended periods of time. This is usually considered as a process of etching and is said to remove the surface recombination centres (Dare-Edwards and Hamnett 1979). However as no simple aquated $\mathrm{Ti}^{4+}$ ion exists because of the high charge-to-ionic radius ratio, no definite hydroxide, $\mathrm{Ti}(\mathrm{OH})_{4}$, is stable and consequently hydrated oxides or basic oxosalts of titanium are formed even in dilute aqueous acids. Thus, species such as $\mathrm{Ti}(\mathrm{OH})_{3}^{+}$or $\mathrm{Ti}(\mathrm{OH})_{2}^{2+}$ are possible which polymerizes to various degrees, giving rise to hydroxylated surfaces. It is, therefore, easy to build up surface states on titanate electrodes in dilute acids. Part of the hydroxyls can be replaced with isoelectronic fluoride ions leading to surface states with higher stability. The influence of these surface states on photon efficiency is presently investigated.

\section{Experimental aspects}

Details regarding the preparation of polycrystalline titanates of alkaline earth metals and of lead $\left(\mathrm{MTiO}_{3}\right.$ where $\mathrm{M}=\mathrm{Ba}, \mathrm{Sr}, \mathrm{Ca}$ or $\mathrm{Pb}$ ) with high purity, sintering of their solid solutions into high density ceramics and characterization of the different phases 
were the same as given in the earlier publications (Kutty and Gomathi Devi 1985, 1986). $\mathrm{Ba}\left(\mathrm{Ti}, \mathrm{ZrOO}_{3}\right.$ solid solutions were prepared by the hydrothermal method (Vivekanandan et al 1987). Sinter density of all the specimens ranges from 94 to $98.5 \%$, with an average grain size of $25-40 \mu \mathrm{m}$. All the electrodes were annealed at $p_{\mathrm{O}_{2}}=10^{-10}-10^{-22} \mathrm{~atm}$ and $1000 \mathrm{C}$ for $3 \mathrm{~h}$ which resulted in resistivities of 2 $378 \mathrm{ohm}$. cm. Because of the low transition metal impurity contents (total $<40 \mathrm{ppm}$ ), higher conductivity for $\mathrm{SrTiO}_{3}$ electrodes could be achieved under the same annealing conditions. ( $\mathrm{Sr}, \mathrm{Pb}_{3} \mathrm{TiO}_{3}$ with $>35 \mathrm{~mol} \% \mathrm{PbTiO}_{3}$ could not be annealed in atmospheres of low $\mathrm{p}_{\mathrm{O}_{2}}$ due to the formation of metallic lead. $\mathrm{Ba}(\mathrm{Ti}, \mathrm{Zr}) \mathrm{O}_{3}$ samples have to be annealed at $>1350^{\circ} \mathrm{C}$ to obtain the necessary conductivity. The electrode mounting methods and the photoelectrochemical measurements were the same as given earlier (Kutty and Gomathi Devi 1985).

When as-annealed electrodes were used. care was taken to protect the front surface during contact making and mounting. Otherwise, the electrode surface was exposed to dilute acids in such a way that deep etch pits are not formed whereas relief on the grain boundaries can be observed in SEM studies. Maximum enhancement in photocurrent was obtained when the electrodes were exposed to mixtures of dilute $\mathrm{HNO}_{3}+\mathrm{HF}$ at $80.95 \mathrm{C}$ for $60-80 \mathrm{~min}$. Surface compositional changes of the electrodes were studied using X-ray photoelectron spectroscopy (XPS) using a PEI model 568 ESCA/Auger System with monochromatic $\mathrm{MgK} x \mathrm{X}$-ray source. Initially the survey-scans were recorded to identify the elements on the surface followed by data acquisition over a narrow energy range of $25 \mathrm{eV}$. The spectra were signalaveraged on a Nova 900 mini computer. Sputter-etching of the samples was carried out with a defocussed Ar-ion beam source. All the bands were referenced to $C(1 s)$ peak at $285.0 \mathrm{eV}$. The standard samples used for intensity calibrations were pressedpellets of $\mathrm{TiO}_{2}$, TiO or Ti metal foil as well as single crystals of $\mathrm{TiO}_{2}$ and $\mathrm{SrTiO}_{3}$.

\section{Results}

The temperature dependence of dielectric constant for some of the presently prepared perovskite phases before annealing in atmospheres of low $p_{\mathrm{O}_{2}}$ is shown in figure 1. The maxima observed at the Curie points for $\mathrm{BaTiO}_{3}$ are broad in the case of polycrystalline samples. The $\varepsilon$-maximum shifts to lower temperatures with $\mathrm{Sr}$ content in $(\mathrm{Ba}, \mathrm{Sr}) \mathrm{TiO}_{3}$ as well as in $(\mathrm{Pb}, \mathrm{Sr}) \mathrm{TiO}_{3}$ so that higher $\varepsilon$ 's are obtained at room temperature. This is true for $\mathrm{Ba}(\mathrm{Ti}, \mathrm{Zr}) \mathrm{O}_{3}$ as well, with the difference that $\varepsilon$ maxima of the three transition points originally present in $\mathrm{BaTiO}_{3}$ move closer, leading to strong and broad maximum, as shown in figure 1. These abnormal characteristics are explained on the basis of diffuse phase transitions prevailing in perovskite phases (Smolenskii 1970; Hennings et al 1982).

Variations in photocurrent $\left(I_{p}=I_{\mathrm{ill}}-I_{\text {dark }}\right)$ with the applied voltage for photoanodes of $(\mathrm{Ba}, \mathrm{Sr}) \mathrm{TiO}_{3}$ solid solutions are given in figure 2 . The as-annealed specimens, without further surface treatments, show lower photocurrents than when the same electrodes are surface-etched in conc. $\mathrm{H}_{2} \mathrm{SO}_{4}$ at $210^{\circ} \mathrm{C}$. It is evident from figure 2 that the photoresponse does not show any correlation with the dielectric constant for neither the as-annealed nor the surface etched electrodes. Similar is the behaviour of $(\mathrm{Sr}, \mathrm{Pb}) \mathrm{TiO}_{3}$ photoanodes as shown in figure 3. $\left(\mathrm{Sr}_{0.9} \mathrm{~Pb}_{0.1}\right) \mathrm{TiO}_{3}$ with $\varepsilon$ lower than that of $\left(\mathrm{Sr}_{0.7} \mathrm{~Pb}_{0.3}\right) \mathrm{TiO}_{3}$ at room temperature has higher photocurrents. The non-correlative behaviour of photoresponse with the dielectric constant becomes 


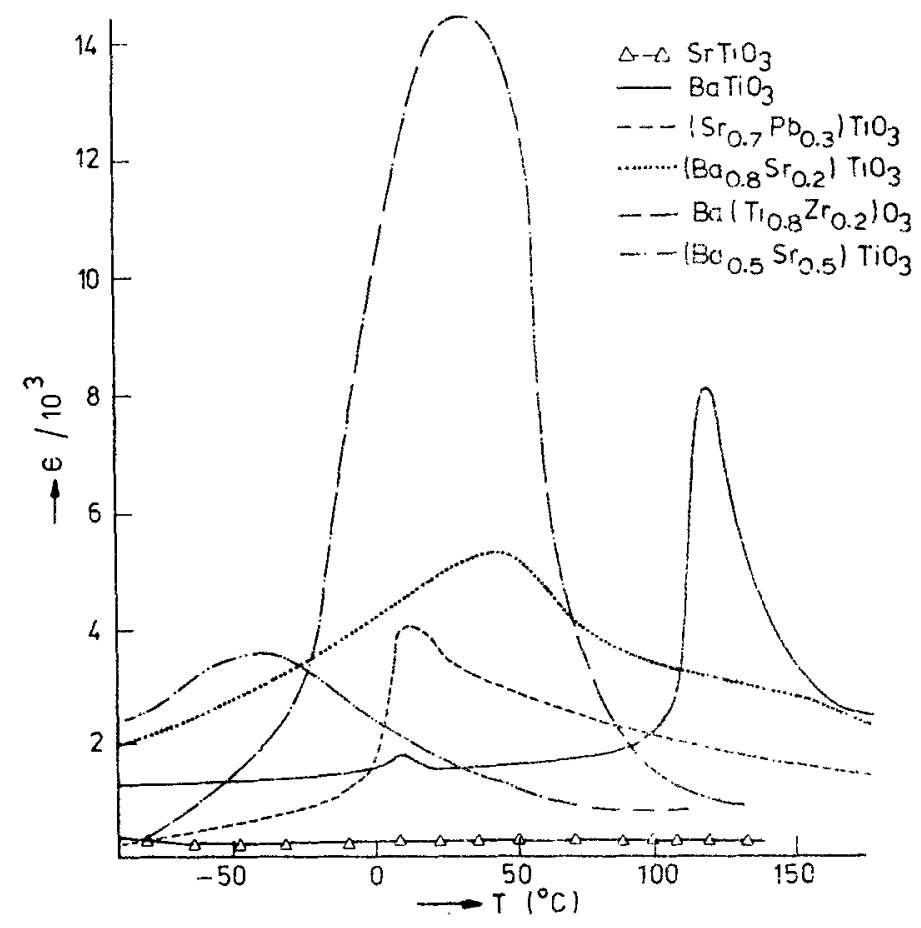

Figure 1. Temperature dependence of dielectric constant of sintered polycrystalline ceramics of perovskite titanates.

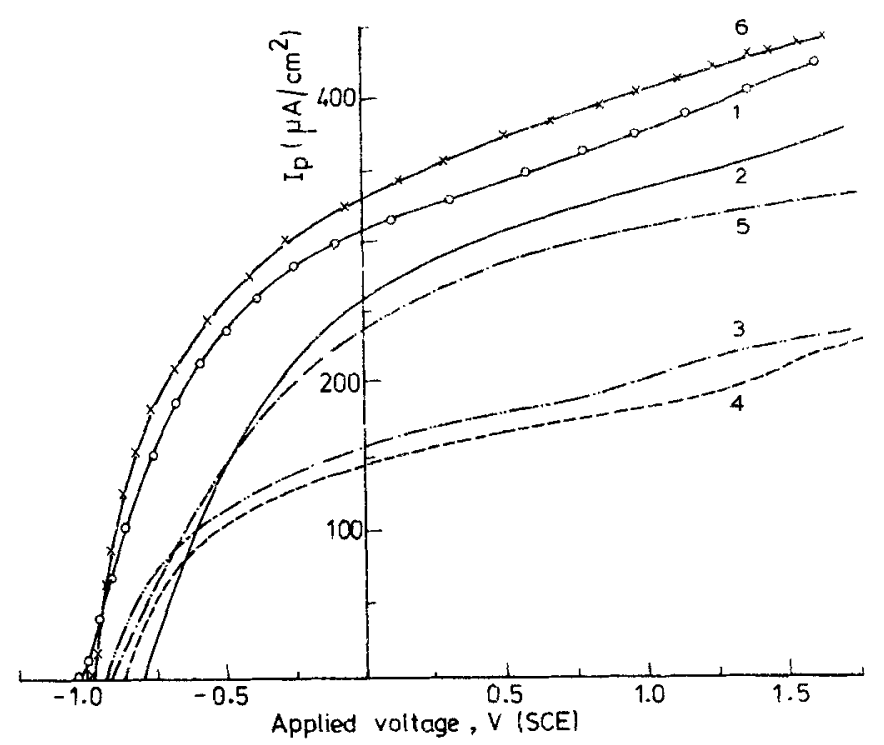

Figure 2. Variation in photocurrent with applied voltage for semiconductor electrodes of (1) $\mathrm{SrTiO}_{3}$, (2) $\mathrm{BaTiO}_{3},(3)\left(\mathrm{Ba}_{0.5} \mathrm{Sr}_{0.5}\right) \mathrm{TiO}_{3}$, (4) $\left(\mathrm{Ba}_{0.8} \mathrm{Sr}_{0.2}\right) \mathrm{TiO}_{3}$, as-annealed at $1000^{\circ} \mathrm{C}$ in an atmosphere of $p_{\mathrm{O}_{2}} \simeq 1 \times 10^{-22} \mathrm{~atm}$. (5) and (6) are the same as in (3) and (4) respectively, after surface treatment in conc. $\mathrm{H}_{2} \mathrm{SO}_{4}$ at $210^{\circ} \mathrm{C}$. Illumination: $\lambda=350-400 \mathrm{~nm}$, $I_{0}=10.2 \mathrm{~mW} / \mathrm{cm}^{2}$. 


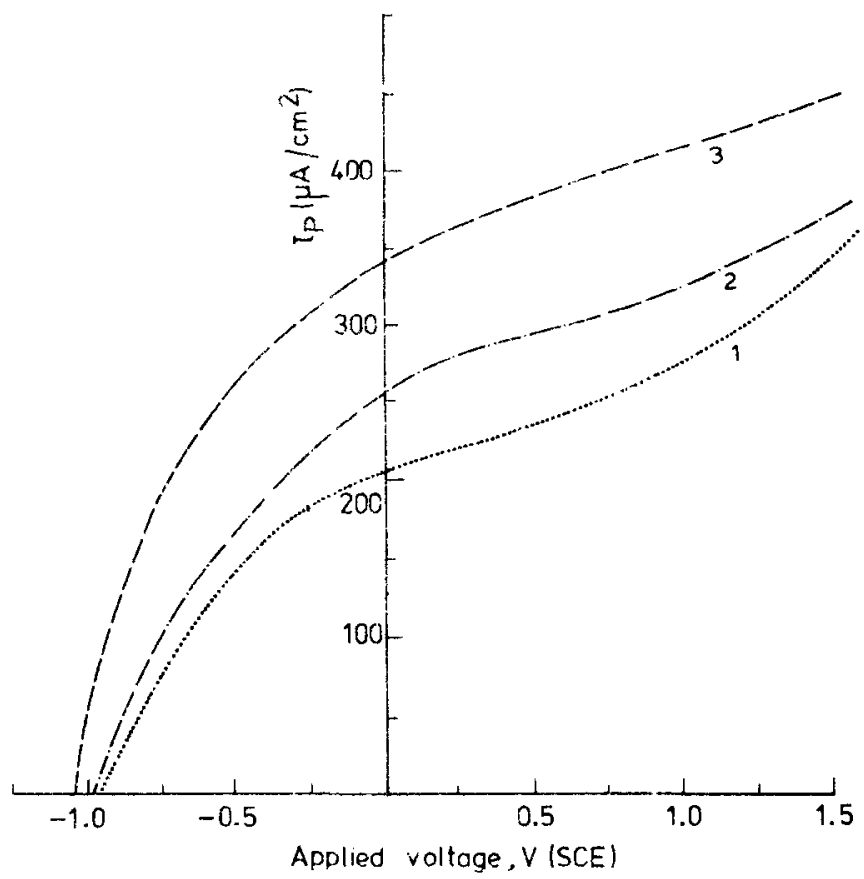

Figure 3. Photocurrent-apflied voltage relations for the as-annealed electrodes of (1) $\left.\left(\mathrm{Sr}_{0,6} \mathrm{~Pb}_{6,1}\right) \mathrm{TiO}_{3}(2)\left(\mathrm{Sr}_{4}\right)-\mathrm{Ph},{ }_{3}\right) \mathrm{TiO}_{3}\{3\}$ is the same as in (1) after surface treatment in $8 \mathrm{M}$ $\mathrm{HNO}_{3}$ at $60 \mathrm{C}$.

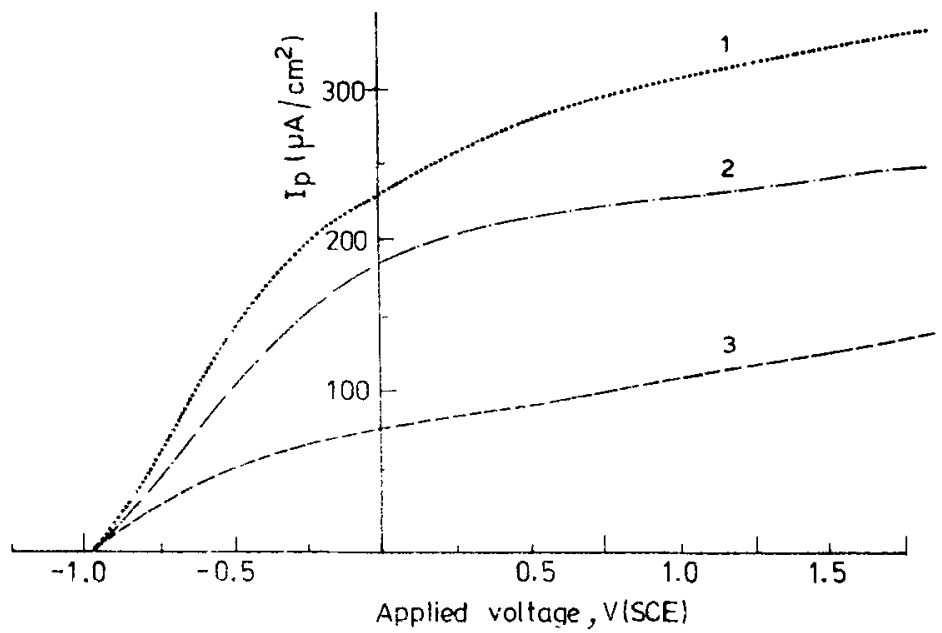

Figure 4. Photwcurrent-applied voltage curves for as-annealed electrodes of (1) $\mathrm{Ba}\left(\mathrm{Ti}_{0,5} \mathrm{Zr}_{6,0}\right) \mathrm{O}_{3}$, (2) $\mathrm{Ba}\left(\mathrm{Ti}_{4}, \mathrm{Zr}_{1,}, \mathrm{O}_{3}\right.$ and (3) $\mathrm{Ba}\left(\mathrm{Ti}_{n 8} \mathrm{Zr}_{0 \cdot 2}\right) \mathrm{O}_{3}$.

more cvident for $\mathrm{Ba}(\mathrm{Ti}, \mathrm{Zr}) \mathrm{O}_{3}$ electrodes (figure 4). In this case, photocurrents are lower for electrodes with higher Zr-contents and can be attributed to lower $N_{D}$ values, which leads to high ohmic potential drop (IR) within the semiconductor. According to equation (3). decreasing $N_{D}$ values ought to have enhanced the width of the depletion layer and hence the photocurrent. Since this relation is limited by the 
IR drop within the semiconductor, the overall cell efficiency decreases. There may be an optimum charge carrier concentration at which IR drop is not dominant, yet larger depletion layer width can be maintained. However such a situation could not be experimentally achieved, as seen from the results of $\left(\mathrm{Ba}_{0.7} \mathrm{Sr}_{0.3}\right) \mathrm{TiO}_{3}$ electrodes (figure 5). In this case, the specimens with different $N_{D}$ values have been prepared by varying the annealing conditions i.e. atmosphere of different $p_{\mathrm{O}_{2}}$ and higher temperatures. Bulk $N_{D}$ values of these samples are calculated from the conductivity as well as Hall mobility data obtained by the Van der Pauw technique. $N_{D}$ values at the depletion layer are obtained from the Mott-Schottky plots for the same set of electrodes in $1 \mathrm{M} \mathrm{NaOH}$ by a bridge technique at $1 \mathrm{kHz}$. The latter values are somewhat larger than those of the bulk measurements. Figure 5 shows that photocurrents at $V_{\text {appl }}=\mathrm{O}(\mathrm{SCE})$ do not decrease with $N_{D}$ whereas the photocurrents reach an apparent saturation. Considering the IR drops at low $N_{D}$ 's, the curves in figure 5 ought to have gone through a maximum if equation (3) has been valid.

Photon efficiencies of titanate electrodes have been investigated as a function of surface treatment conditions in various acids of different concentrations. Better results are obtained in dilute acid mixtures of $\mathrm{HNO}_{3}+\mathrm{HF}$. Photocurrent increases with time of exposure to this reagent at $80-95^{\circ} \mathrm{C}$ and reaches the maximum within 60-80 min. The treatment shifts the onset photopotentials to negative values. $I_{n}-V_{\text {(appl) }}$ curves become steeper (figure 6) and the limiting photocurrent is enhanced by nearly one order of magnitude. Longer exposure to the same medium somewhat lowers the photoresponse and is accompanied by the formation of deeper etch pits as revealed by SEM. The thickness of the layer removed can be approximated to $<0.3 \mu \mathrm{m}$ in $60 \mathrm{~min}$, from the concentration of $\mathrm{M}^{2+}$ and $\mathrm{Ti}^{4+}$ in the aqueous medium (AAS method). Although the treatment of the titanate electrode surfaces with acids is considered as a simple process of dissolution, the present results show that the treatment produces complex surface effects. It is evident from figure 6 that surface modifications have pronounced influence on the photoresponse as compared to

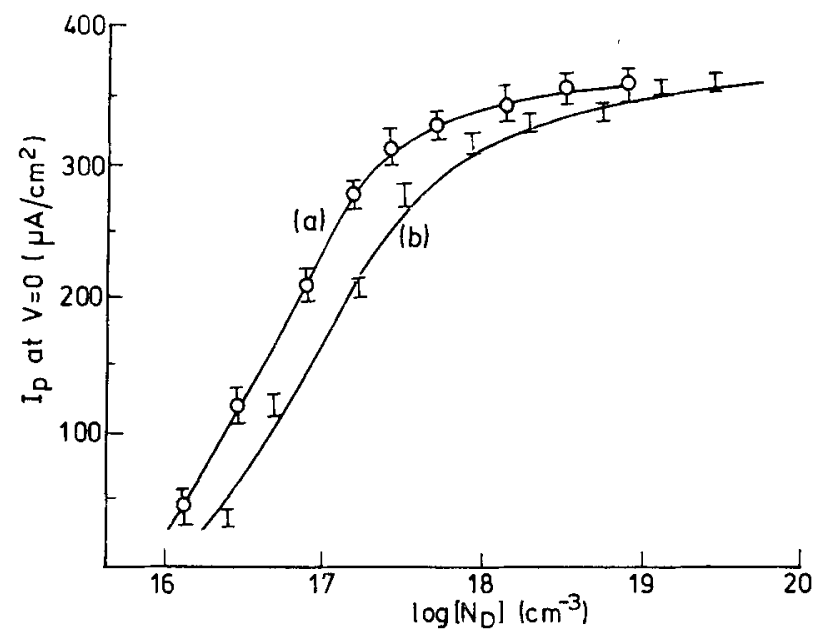

Figure 5. Variation in photocurrent with charge carrier density for $\left(\mathrm{Ba}_{0.7} \mathrm{Sr}_{0.3}\right) \mathrm{TiO}_{3}$ electrodes annealed in the atmospheres of $p_{\mathrm{O}_{2}}$ varying from $1 \times 10^{-9}$ to $1 \times 10^{-23} \mathrm{~atm}$ at $1000^{\circ} \mathrm{C}$. (a) bulk $N_{D}$ obtained from conductivity and Hall mobility data, (b) $N_{D}$ at the depletion layer from Mott-Schottky plots. $V_{\text {appl }}=0 \mathrm{~V}(\mathrm{SCE})$. 


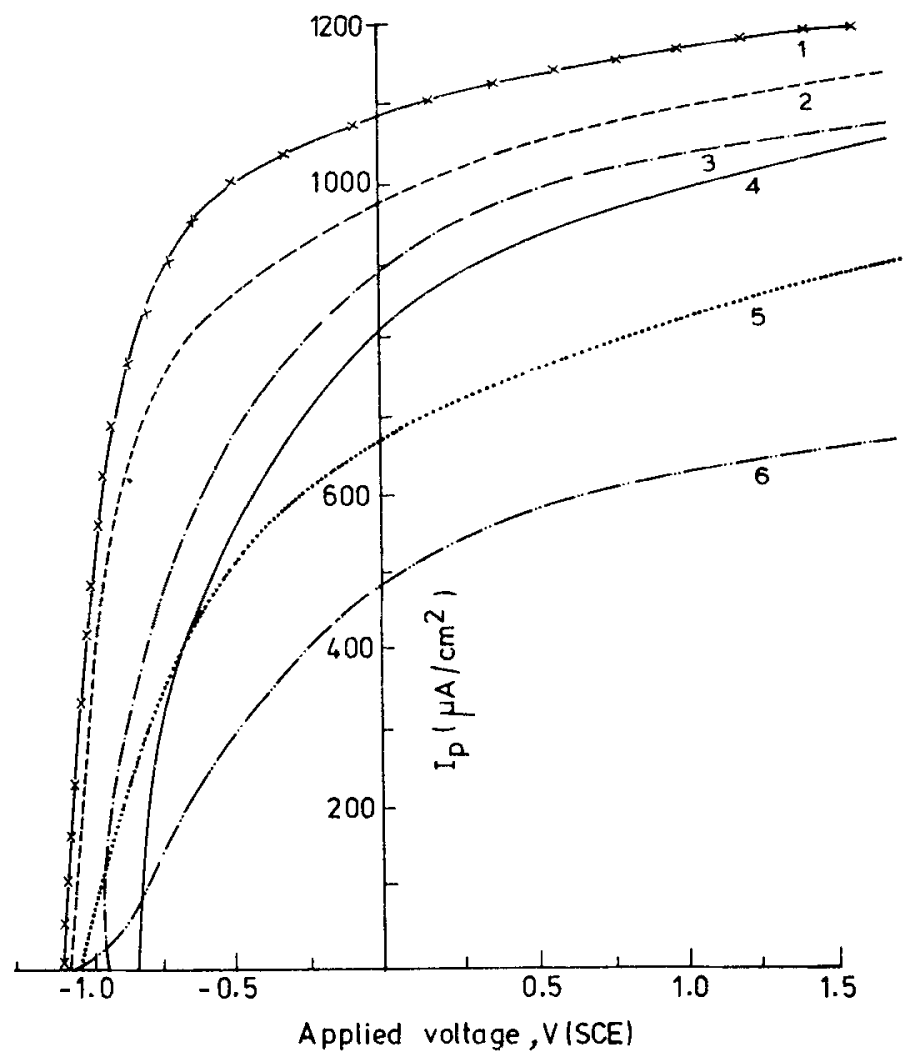

Figure 6. Photocurrent vs applied voltage for perovskite electrodes: (1) $\left(\mathrm{Sr}_{0.9} \mathrm{Ba}_{0.1}\right) \mathrm{TiO}_{3}$, (2) $\mathrm{SrTiO}_{3}$. (3) $\left(\mathrm{Sr}_{0,7} \mathrm{~Pb}_{0.3}\right) \mathrm{TiO}_{3}$, (4) $\mathrm{BaTiO}_{3},(5) \mathrm{Ba}\left(\mathrm{Ti}_{0,5} \mathrm{Zr}_{0.05}\right) \mathrm{O}_{3}$ and (6) $\mathrm{Ba}\left(\mathrm{Ti}_{0.9} \mathrm{Zr}_{0.1}\right) \mathrm{O}_{3}$ Surface treated in $0.5 \mathrm{M} \mathrm{HNO}_{3}+05 \mathrm{M} \mathrm{HF}$ at $85^{\circ} \mathrm{C}$ for $80 \mathrm{~min}$.

altering the depletion layer width by varying the bulk as well as $N_{D}$ values. MottSchottky plots of the surface-treated electrodes show marginal decrease of $N_{D}$ within the depletion layer when compared to the corresponding as-annealed specimens. The influence of dilute $\mathrm{HNO}_{3}$ and separately dilute $\mathrm{HF}$ has been $<30 \%$ enhancement over the responses of the as-annealed electrodes. The possible effect of fluoride ions in the electrolyte is found negligible since the addition of $\mathrm{NaF}, \mathrm{Na}_{2} \mathrm{TiF}_{6}$ or $\mathrm{TiOF}_{2}$ to $1 \mathrm{M} \mathrm{NaOH}$ does not show any changes in photocurrents. Further, the effective hole scavengers such as EDTA, TEOA, acetate, sulphite or hypophosphite, when added to the electrolyte, have little effect on the photocurrents of the as-annealed as well as surface-treated electrodes. Stability of the electrodes exposed to dilute acid is investigated for extended periods upto $\sim 180 \mathrm{~h}$ of operation. If preserved under $\mathrm{NaOH}$ electrolyte the photocurrents remain unchanged. If the surface-treated electrodes are preserved under inert atmosphere in a container, the photocurrent slowly diminishes whereas on preserving in air, the decrease is faster.

Wavelength dependence of photoresponse of titanate electrodes after surface treatment in dilute acids has been studied. Figure 7 a shows the variation in photon efficiency normalized to the same intensity of radiation with wavelength. The treated electrode shows the red shift in photoresponse as compared to the untreated specimens. These spectral changes are comparable to those observed for the bulk 


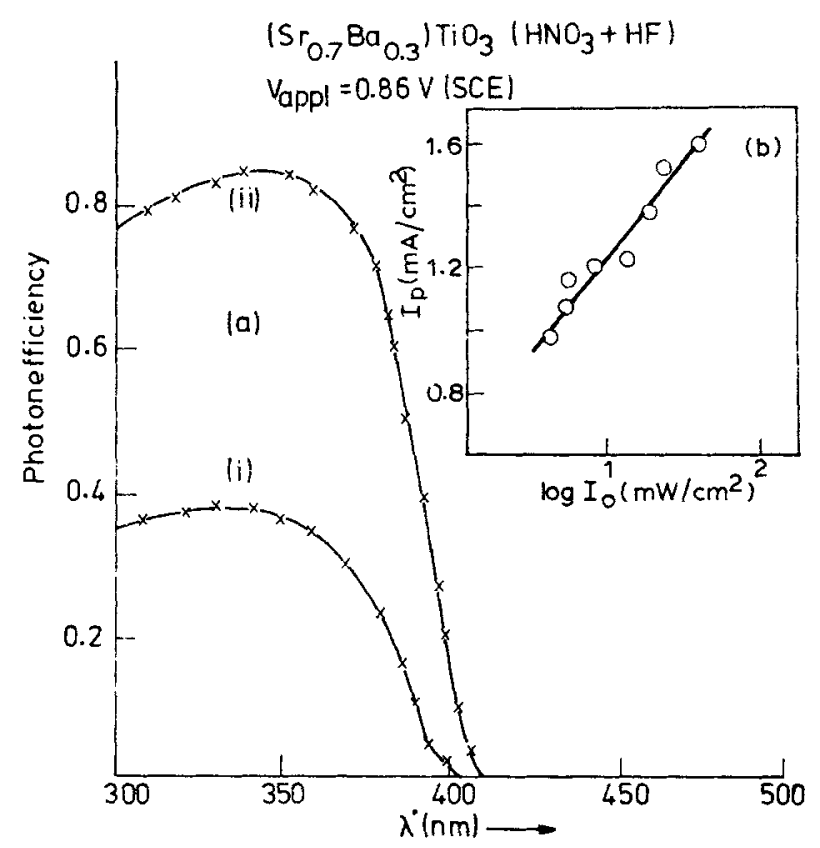

Figure 7. (a) Variation of photon efficiency with wavelength for (i) as-annealed and (ii) surface treated (as in figure 6) electrodes of $\left(\mathrm{Sr}_{0.7} \mathrm{Ba}_{0.3}\right) \mathrm{TiO}_{3} . V_{\text {appl }}=0.86 \mathrm{~V}$ (SCE), $I_{0}=21 \mathrm{~mW} / \mathrm{cm}^{2}$. (b) Change in photocurrent with intensity of light for the same electrode as in (a) at $V_{\text {ippi }}=1.0 \mathrm{~V}(\mathrm{SCE})$.

fluorinated $\mathrm{TiO}_{2}$ electrodes (Wold and Dwight 1980). The treatment modifies the depletion layer towards the surface region (space charge region) is further demonstrated from the fact that variations in light intensity proportionally alter the photocurrent at constant applied potential (figure 7 b).

Surface compositions of the titanate electrodes. treated in dilute acids have been investigated by XPS. The results from $\mathrm{SrTiO}_{3}$ are presented as a typical example. The $\mathrm{O}(1 s)$ band of $\mathrm{TiO}_{2}$ as well as of $\mathrm{SrTiO}_{3}$ appears with the same binding energy (BE) of $530.2 \mathrm{eV}$. This band remains unchanged with annealing in low $p_{\mathrm{O}_{2}}$ atmosphere at elevated temperatures and also after mechanically polishing the electrode surface or on Ar-ion sputter etching. Whereas Ti $\left(2 p_{1 / 2,3 / 2}\right)$ bands shift continuously to lower energies with the extent of chemical reduction so that the difference, $\triangle \mathrm{BE}$ $=\mathrm{O}(1 s)-\mathrm{Ti}\left(2 p_{3 / 2}\right)$ increases from 71.0 to $73.6 \mathrm{eV}$ for $\mathrm{SrTiO}_{3}$. This is accompanied by the decrease in $N_{\mathrm{O}} / N_{\mathrm{Ti}}$ ratio from 3.0 to $2 \cdot 7$. Although existence of both Ti(III) and $\mathrm{Ti}(\mathrm{IV})$ species can be expected in chemically-reduced $\mathrm{TiO}_{2}$ and $\mathrm{SrTiO}_{3}$, no multiplicity in $\mathrm{Ti}\left(2 p_{1 / 2,3 / 2}\right)$ bands is noticed (figure 8$)$. Since a maximum value of $\triangle \mathrm{BE}(=76.5 \mathrm{eV})$ is observed for TiH films and Ti-metal foils, the shift in Ti-bands to lower energies corresponds to electron enrichment in the system. When $\mathrm{SrTiO}_{3}$ electrode surface is exposed to $0.5 \mathrm{M} \mathrm{HNO}_{3}$ for $80 \mathrm{~min}$ at $90^{\circ} \mathrm{C}$, a form of oxygen with higher binding energy is noticed with $\mathrm{O}(1 \mathrm{~s})=532.5 \mathrm{eV}$ and $\sim 20 \%$ peak area of the main $O(1 s)$ band. This side band disappears on Ar-ion sputter etching to a depth of $\sim 150 \AA$ and therefore corresponds to the presence of the hydroxyl groups or strongly chemisorbed water on the surface. Another feature is the multiplicity of $\mathrm{Ti}\left(2 p_{1 / 2,3 / 2}\right)$ bands with characteristics corresponding to both chemically reduced as 


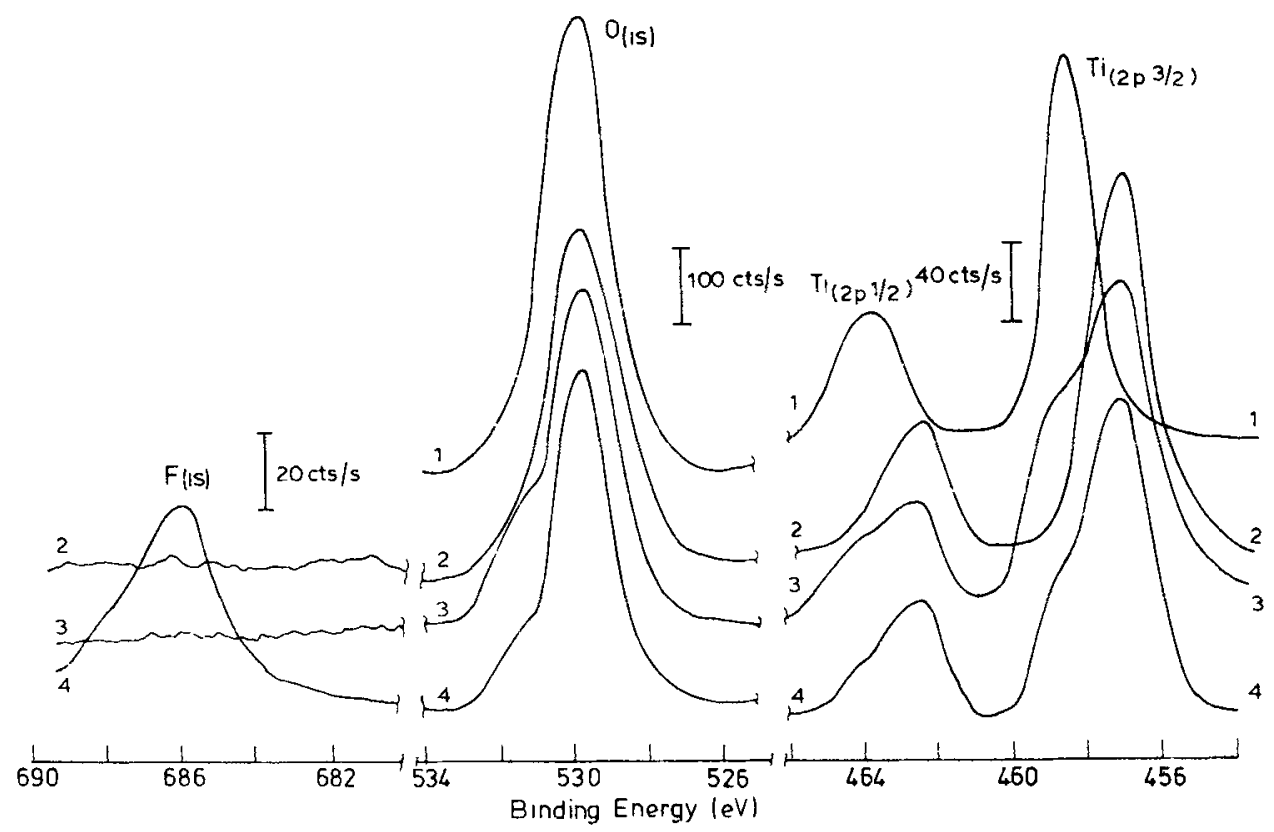

Figure 8. X-ray photoelectron spectra of $\mathrm{SrTiO}_{3}$ electrodes (1) chemically unreduced specimen; (2) reduced at $1000^{\circ} \mathrm{C}$ in $n_{\mathrm{O}_{2}} \simeq 10^{-23} \mathrm{~atm}$ (3) electrode exposed to $0.5 \mathrm{M} \mathrm{HNO}_{3}$ at $90^{\circ} \mathrm{C}$ for $80 \mathrm{~min}$; (4) electrode exposed to $0.5 \mathrm{M} \mathrm{HNO}_{3}+0.05 \mathrm{M} \mathrm{HF}$ at the same conditions as in (3).

well as the unreduced $\mathrm{SrTiO}_{3}$. Since multiplicity in these bands is not observed for the as-annealed samples, the above results can only be explained on the basis of individual surface regions with the above features within $6 \mathrm{~mm}^{2}$ spot area of the Xray beam falling on the electrode surface during XPS probe. This is further supported by the fact that Ar-ion sputter etching changes the spectrum from figure $8 \cdot 3$ to $8 \cdot 2$. Besides, $N_{\mathrm{O}} / N_{\mathrm{Tj}}(=2.9)$ is higher than that of the as-annealed surfaces. Neither the band position nor the line width of $\operatorname{Sr}\left(2 p_{3 / 2}\right)$ with $\mathrm{BE}=270 \cdot 2 \mathrm{eV}$ changes during the high temperature annealing. Figure 8.4 gives the $\mathrm{O}(1 s)$ and $\mathrm{Ti}\left(2 p_{1 / 2,3 / 2}\right)$ bands for the electrode surface exposed to $0.5 \mathrm{M} \mathrm{HNO}_{3}+0.05 \mathrm{M} \mathrm{HF}$. The presence of fluoride on the surface is evident from the appearance of $\boldsymbol{F}(1 s)$ band. The persistence of surface hydroxyls is indicated from the higher energy $\mathrm{O}(1 s)$ side band with low intensity. Another characteristic feature is the corresponding decrease in intensities of Ti $\left(2 p_{1 / 2,3 / 2}\right)$ higher energy side bands. These surface features disappear on Ar-ion sputter etching to a depth of $\gtrsim 80 \AA$. The dominance of the chemically reduced form of $\mathrm{SrTiO}_{3}$, as indicated from $\mathrm{Ti}\left(2 p_{1 / 2,3 / 2}\right)$ bands, can be explained on the basis of aliovalent anion compensation such as $\mathrm{Sr} \mathrm{Ti}_{-x}^{\mathrm{IV}} \mathrm{Ti}_{x}^{\mathrm{III}} \mathrm{O}_{3-x} \mathrm{~F}_{x}$ so that oxygen vacancies are minimized, yet maintaining higher charge carrier concentration as envisaged by Wold and coworkers (Derrington et al 1978; Wold and Dwight 1980) for $\mathrm{WO}_{3-x} \mathrm{~F}_{x}$ and $\mathrm{TiO}_{2-x} \mathrm{~F}_{x}$ bulk phases. Thus the XPS data indicate that the titanate electrodes exposed to dilute $\mathrm{HNO}_{3}+\mathrm{HF}$ for extended periods of time have heterogeneous surface regions with respect to chemical characteristics. However from our limited XPS experiments, neither the dimensions nor magnitude of spatial separation of these inhomogeneous regions could be evaluated. 


\section{Discussion}

The results show the importance of surface treatment in enhancing the photoresponse. The role of surface states as mediators of interfacial charge transfer process is often ambiguously discussed. Theoretically, surface states are essential because the exchange of charges between electronic states in the semiconductor and the appropriately located redox energy levels within the electrolyte is possible only if the energy difference is $\sim k_{B} T$, following Frank-Condon principle (Gerischer 1970). At the same time, surface states are said to decrease the efficiency of the photoelectrodes by acting as recombination centres and by encouraging the dark reactions ( $t$ 'Lam and Franceschetti 1982; Praet et al 1983). It is possible that enhanced photoresponse is not by totally eliminating the surface states but by suitably balancing the losses due to recombination through discriminate tailoring of the surface states. This necessitates identification of all the surface states and understanding the mechanism of their influence on the space charge layer. Although many publications on photoelectrochemistry of perovskite titanate passingly mention about the surface treatment, most of them do not give any details and in no case have attempts been made to characterize the surface composition after electrode treatment. Exposure of the electrode to molten $\mathrm{NaOH}$ or $\mathrm{KHSO}_{4}$ or $\mathrm{H}_{2} \mathrm{SO}_{4}+\left(\mathrm{NH}_{4}\right)_{2} \mathrm{SO}_{4}$ at elevated temperature leads to surface oxidation thereby enhancing IR drop within the semiconductor. However, the changes in $I_{p}-V_{(\text {appl) }}$ curves by way of the precipitous increase in photocurrent nearer $V_{\text {on }}$ have been taken as a sign of removal of surface states and recombination centres (Dare-Edwards and Hamnett 1979; Gorbecht and Gerischer 1979; Laser and Bard 1976).

The enhancement in photocurrent, the cathodic shift of $V_{\text {on }}$, the variation of photoresponse at longer wavelength and light intensity, and the marginal decrease in $N_{D}$ values in the space charge layer during surface exposure to dilute acid can be explained in terms of the elimination of undesirable recombination centres within the space charge layer. The increase in photocurrent during the surface treatment is fairly large that it cannot be attributed to modified geometrical features such as surface roughness which, in any case, is not indicated by SEM observations. The enhanced photocurrents accompanied by the cathodic shift of $V_{\text {on }}$ reflect the elimination of recombination centres that require higher bias potentials. The energy states arising from these recombination centres may be located anywhere within the band gap since they are coupled to the semiconductor lattice sufficiently strongly to allow inelastic capturing of charge carriers. The centres that are located nearer the Fermi level can capture electrons so that the rate of transfer of electrons from the space charge layer to the conduction band is decreased. Alternatively the centres located nearer the valence band can trap the holes so that the rate of hole transfer to the electrolyte is lowered. The electron or hole thus accumulated within the space charge layer prevents further mobility of the respective charge carrier from the diffusion layer that is said to be located just below the space charge layer. If the accumulation of holes is the cause for the lower photoresponse in the untreated electrodes, addition of hole scavengers in the electrolyte ought to have enhanced the photocurrent. Since this is not the case, more probable is the removal of surface states nearer the Fermi level which capture the conduction band electrons at a rate at which they are photogenerated. When these surface states are removed, the electrons find less number of recombination centres and larger photocurrent flows 
through the circuit. The decreasing photoresponse of the surface-treated electrodes during preservation in air as compared to that in $\mathrm{N}_{2}$ or $\mathrm{Ar}$ is also indicative of the prevalence of electron recombination centres, rather than of holes at the surface since the latter must have been stable under such conditions. Our investigations on the defect states in titanates (Kutty et al 1985; Kutty 1986) and the electrochemical studies on polished electrodes (Kutty and Gomathi Devi 1985) have shown that these electron recombination centres are related to oxygen vacancies coupled to adjoining titaniums to form $\mathrm{Ti}^{3+}-V_{\mathrm{O}}$ defect complexes. Hydroxyls or fluoride ions may decrease the concentration of such defect complex by occupying the $V_{0}$ sites within the space charge layer. Increased photoresponse to longer wavelength is proposed to arise from the elimination of oxygen vacancies in $\mathrm{TiO}_{2-x} \mathrm{~F}_{x}$ (Wold and Dwight 1980). Due to higher electronegativity of fluorine than of oxygen, the valence band edge can be expected to be lowered so that the band gap increases. This should lead to the shift in photoresponse to shorter wavelength as well as the lowering of $V_{\mathrm{fb}}$. Both these changes are experimentally observed in the opposite direction, and can be better explained on the basis of the heterogeneous surface layers that leads to non-uniform space charge potential distribution near the electrode surface, depending upon the charge density and mobility of the individual regions (Dutoit et al 1976). The microelectric fields generated from these surface regions may cause potential energy perturbations in the neighbouring regions of the bulk phase so that sharp conduction and valence band edges no longer exist, leading to tailing off of allowed eigenstates into the forbidden gap. This will account for the shift in photoresponse to longer wavelengths which arises from the modifications within the space charge layer rather than in the bulk.

The second possible contribution to the enhanced photoresponse arises from the heterogeneous surface regions differing in chemical composition which can act similar to metal catalyst islands on photocathodes (Heller 1984). There are no reports on the non-metal catalysts on semiconductor electrodes and the case of $\mathrm{NiO} / \mathrm{SrTiO}_{3}$ has been shown to be related to the existence of $\mathrm{Ni}$-metal at the interface (Domen et al 1986). Although the requirements such as electron-hole recombination, charge leakage and barrier height equally apply to photoanodes and the essentiality of the catalysts on photoanodes seems to be relaxed. This is because some of the photoanodes, particularly $\mathrm{TiO}_{2}$ and the titanates, function adequately efficiently even in the absence of the catalyst islands. The surface regions with compositional heterogeneity can alter the height of the potential energy barrier that separate the photogenerated electrons from holes and also lower the rate of recombination at the space charge region. This is due to the local differences in charge carrier density and electron mobility of the heterogeneous surface regions differing in chemical characteristics. Further, there may be local modifications to lighttransmission characteristics arising from the screening of optical electric field by the conduction electrons in those surface regions with higher electron mobility. This leads to the channelling of light into the regions of the substrate (in this case, the bulk of the electrode) between such islands and can be explained on the basis of effective medium theory for the optical response of heterogeneous materials (Bruggemann 1936). Perhaps the non-essentiality of the catalyst islands on titanate photoanodes may be due to the innately formed surface compositional heterogeneity during the variously employed surface treatments. 


\section{Conclusions}

Surface hydroxyls and fluoride ions incorporated within the space charge layers have sufficiently large concentrations to be detected by suitable physical techniques. In the presence of corresponding surface states, the changes in potential at the interface should equally affect the Helmholtz region at the electrolyte side of the interface and modify the charge transfer to include other steps than envisaged in the Schottky barrier model.

\section{Acknowledgements}

Thanks are due to Dr H Rosenstengel, Bielefeld University (F.R.G) for the XPS results. Financial support of the Department of Non-Conventional Energy Sources, Government of India is gratefully acknowledged.

\section{References}

Bockris J O' M and Khan S U M 1985 J. Electrochem. Soc. 1322648

Bruggemann D A G 1936 Ann. Phys. (Leipzig) 25645 and 665

Butler M A 1977 J. Appl. Phys. 481914

Dare-Edwards M F and Hamnett A 1979 J. Electroanal. Chem. Interf. Electrochem. 105283

Derrington C E, Godek W S, Castro A and Wold A 1978 Inorg. Chem. 17977

Domen K, Kudo A, Onishi T, Kosugi N and Kuroda H 1986.J. Phys. Chem. 902992

Dutoit E C, Cardon F and Gomes W P 1976 Ber Bunsgenges Phys. Chem. 80475

Gerischer H 1960 Zeit. Phys. Chem. N.F. 26223

Gerischer H 1970 in Physical chemistry: An advanced treatise (eds) H Eyring, D Henderson and W Jost (New York: Academic Press) Vol. 95 p. 463

Gerischer H and Ekardt W 1983 Appl. Phys. Lett. 43393

Gorbrecht J and Gerischer H 1979 Solar Energy Mater. 2131

Green M 1959 J. Chem. Phys. 31200

Heller A 1984 Science 2231141

Hennings D. Schnell A and Simon G 1982 J. Am. Ceram. Soc. 65539

Khan S U M and Bockris J O' M 1983 J. Phys. Chem. 872599

Kutty T R N 1986 Proc. Indian Acad. Sci. (Chem. Sci.) 96581

Kutty T R N and Gomathi Devi L 1985 Mater. Res. Bull. 20793

Kutty T R N and Gomathi Devi L 1986 Indian J. Technol. 24391

Kutty T R N, Murugaraj P and Gajbhiye N S 1985 Mater. Res. Bull. 20565

Laser D and Bard A J 1976 J. Electrochem. Soc. 1231828

Praet A, Vanden Kerchoue F, Gomes W P and Cardon P 1983 Solar Energy Mater. 7481

Smolenskii G A 1970 J. Phys. Soc. Jpn. 28 (Suppl.) p. 26

t'Lam R U E and Franceschetti D R 1982 Mater. Res. Bull. 171081

Vivekanandan R, Sam Philip and Kutty T R N 1987 Mater. Res. Bull. 2299

Williams F and Nozik A J 1984 Nature (London) 31221

Wold A and Dwight K 1980 in Solid state chemistry-A contemporary overview (eds) L Smith, J Holt, B Milstein and M Robins (Washington: Am. Chem. Soc.) Adv. Chem. Ser. No. 186 p. 161 